\title{
GRECO: Graphical Electromagnetic Computing for RCS Prediction in Real Time*
}

\author{
Juan M. Rius, M. Ferrando, and L. Jofre \\ Antennas, Microwave and Radar Group \\ Dpt. Teoria del Senyal i Comunicacions \\ Universitat Politècnica de Catalunya \\ Apdo. 30002 \\ 08080 Barcelona \\ Spain
}

\begin{abstract}
T

his paper presents a new and original approach to computing the high-frequency radar cross section (RCS) of complex radar targets in real time, using a 3D graphics workstation. The target (typically, an aircraft) is modeled with the I-DEAS solid-modeling software, using a parametric-surface approach. The high-frequency RCS is obtained through Physical Optics (PO), Method of Equivalent Currents (MEC), Physical Theory of Diffraction (PTD), and Impedance Boundary Condition (IBC) techniques.
\end{abstract}

This method is based on a new and original implementation of high-frequency techniques, which we have called "Graphical Electromagnetic Computing (GRECO)." A graphical-processing approach to an image of the target on the workstation screen is used to identify the surfaces of the target, visible from the radar viewpoint, and to obtain the unit normal at each point of these surfaces. High-frequency approximations to RCS prediction are then easily computed from the knowledge of the unit normal at the illuminated surfaces of the target.

The image of the target on the workstation screen, to be processed by $G R E C O$, is obtained, in real time, from an I-DEAS geometric model, using the 3D graphics hardware accelerator of the workstation. Therefore, the CPU time for the RCS prediction is spent only on the electromagnetic part of the computation, while the more time-consuming geometric-model manipulations are left to the graphics hardware. This hybrid, graphic-electromagnetic computing $(G R E C O)$ results in real-time RCS prediction for complex radar targets.

\section{Introduction}

The aim of this paper is focused on the prediction of the monostatic radar cross section (RCS) of large and complex radar targets. The main objective of the algorithms presented here is obtaining real-time results for arbitrary and very general target shapes, using a graphics workstation. Thus, the RCS prediction software can be easily integrated with the computer-aided-design (CAD) package used to model the target, providing an efficient tool for interactive modeling, design, and analysis of aircraft with RCS specifications.

The computation of the RCS of large and complex targets involves different scattering mechanisms, such as specular reflec-

*[Editor's note: This paper is a companion to a paper entitled, "High-Frequency RCS of Complex Radar Targets in Real Time," which will appear in IEEE Trans. Ant. Prop. See the Editor's Comments in this issue for more information.] tion, diffraction at edges, multiple scattering, shadowing effects, surface-wave scattering at discontinuities, creeping waves, etc. Numerical techniques based on rigorous formulation take into account all these effects, giving very accurate results, but, on the other hand, the computational cost is prohibitive, for very large targets.

Fortunately, it is well known that high-frequency scattering is a local phenomenon, and that the main contributions come from the specular points at surfaces or edges [1]. Accordingly, second-order effects are not usually taken into account, and the RCS of complex targets can be predicted, with reasonable accuracy, by highfrequency asymptotic approximations. Surface reflection is analyzed by Physical Optics. Edge diffraction is analyzed by the Method of Equivalent Currents, in which the far field is computed as radiated by equivalent surface or edge currents, respectively

The main difficulty for predicting the RCS of complex targets, using high-frequency techniques, is the computation of surface and line integrals over an arbitrary shape. This shape is defined by CAD geometric-modeling software, using either a facets-and-wedges approximation, or a parametric-surface approach. In both cases, the unit normal to the surface at each point of the geometric model must be obtained, in order to perform the electromagnetic computations.

An additional problem is that, according to high-frequency theory, these equivalent currents are assumed to be zero over the surfaces or edges not illuminated by the incident wave (i.e., features shadowed by other parts of the aircraft), so that the surface- or edge-radiation integrals extend only over the region of the target illuminated by the incident field.

The classical high-frequency techniques for RCS prediction are based on a target modeled in terms of facets and wedges [2-4] High-frequency approximations can be applied separately to each facet and wedge, so that a closed formula is obtained for Physical Optics [5] and for the Method of Equivalent Currents [1]. The RCS of the whole target is then computed, adding coherently the separate contributions of all the illuminated facets and wedges. Multiple interactions between facets and/or wedges can also be considered by the classical methods.

Further processing is thus required in order to remove the contribution of shadowed parts. The identification of illuminated and shadowed regions, on the geometrical model of the target, is a difficult and very time-consuming problem. Therefore, the classical high-frequency codes must dedicate great effort to the manipulation of the aircraft geometric model, prior to the electromagnetic computations. 
The computational cost required for the identification of shadowed regions and for multiple-scattering computations increases very rapidly with the number of facets required to model a complex radar target. For that reason, the classical techniques are usually implemented on powerful computers, different from the graphics workstations used to model the target. Accordingly, a different approach to compute high-frequency approximations is needed, in order to obtain real-time results on the graphics workstations.

\section{Graphical electromagnetic computing (GRECO)}

These difficulties are overcome by the new graphical processing technique: using a graphics workstation, we can obtain a 3D image of the target (for example, the image generated by the CAD geometric modeling software). If the viewpoint of the target is located at the position of the monostatic radar, then the picture on the workstation screen contains only the illuminated surfaces and edges: the shadowed ones are not visible from the observer's viewpoint, because they have been removed by the $3 \mathrm{D}$ visualization hardware.

Furthermore, if the image is obtained using 3D illumination and rendering, we can define the illumination-source parameters in such a way that the red, green, and blue (R,G,B) components of the color of each pixel of the image are equal to the $\left(n_{x}, n_{y}, n_{z}\right)$ components of the unit vector normal to the surface at this point. The screen memory of the workstation then has six-dimensional information at each pixel: $x, y, z$ coordinates, and the R, G,B color components, which are equal to $\left(n_{x}, n_{y}, n_{z}\right)$. Thus, the screen memory has available all the information needed in order to compute highfrequency approximations. This information is provided in real time by the hardware graphics accelerator of the workstation.

Accordingly, the RCS prediction problem has been reduced to only the electromagnetic part of the computation, which involves the high-frequency surface and line integrals. This is performed processing the $x, y, z, n_{x}, n_{y}, n_{z}$ information of the illuminated surfaces of the target on the workstation screen.

Fresnel reflection coefficients for each pixel can be obtained, because the unit normal to the surface is known $[20,21]$. The PO surface integral is then computed by adding coherently the contribution of each pixel. In order to perform the MEC line integral, the edges are identified as discontinuities of the surface unit normal. We can compute the wedge interior angle, and the edge orientation relative to the observer, because we know the unit normal at both sides of the edge. Thus, we can obtain incremental length diffraction coefficients (ILDCs) for each pixel of the image that lays on an edge, and we can compute the MEC line integral by adding coherently the ILDC contributions.

If the workstation has a hardware graphics accelerator, the image of the target and the $x, y, z, n_{x}, n_{y}, n_{z}$ information for each pixel can be obtained in real time. In conclusion, the CPU running time involves only the electromagnetic part of the computation, leaving the geometric part to the graphics hardware.

As shown in Figure 1, the RCS is obtained by the GRECO code in the following steps [6-11]:

1. Geometric modeling of the aircraft with a $\mathrm{CAD}$ package

2. The image of the target on the workstation screen is obtained in real-time by the graphics hardware accelerator

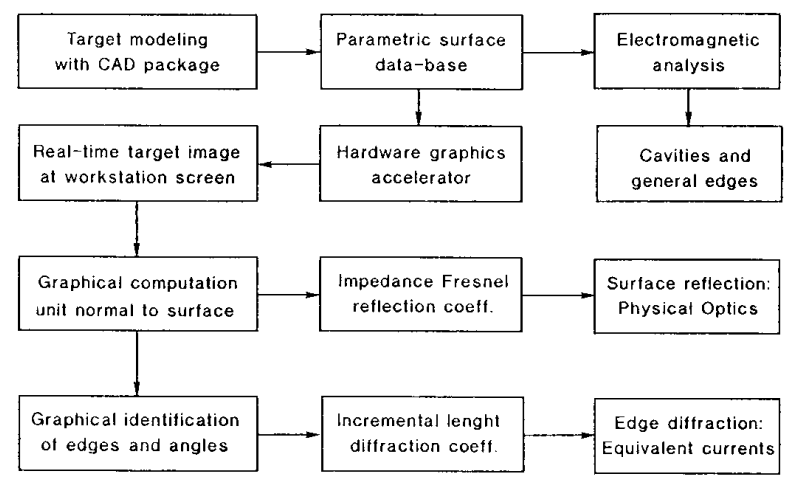

Figure 1. A block diagram with the different steps for RCS prediction by the Graphics Electromagnetic Computing (GRECO) code.

3. The $x, y, z, n_{x}, n_{y}, n_{z}$ coordinates of each point of the illuminated surface are obtained by graphical processing of the image

4. The following high-frequency approximations are then computed:

a. Reflection at perfectly-conducting surfaces by the physical-optics approximation [1]

b. Reflection at coated surfaces by physical-optics and impedance-boundary-condition approximations $[1,20,21]$

c. Diffraction at edges by the method of equivalent currents, using PTD [1] incremental length diffraction coefficients [22]. In the near future, Mitzner ILDCs will be implemented for bistatic RCS prediction [1].

Steps 1 to 3 involve the geometric and graphical part of the computation, and will be explained in detail in Section 3, while Step 4 , the electromagnetic part, will be treated more briefly in Section 4 .

\section{Graphical processing of an image of the target}

\section{1 Target geometric modeling}

A computer-aided-design package for geometric modeling of solids [12] has been used for modeling the target geometry. The aircraft is described either as a collection of facets and wedges, or by parametric surfaces. These last are defined using two-dimensional non-uniform rational B-splines (NURBS) $[13,14]$.

As stated before, classical RCS analysis packages usually describe the target in terms of facets and wedges [2-4]. However, parametric surfaces present the following advantages for both complex-object modeling and RCS prediction and optimization:

- Complex objects require a very large number of facets, while only a few parametric surfaces. Thus, the parametric approach requires a smaller quantity of information to define the model, which results in less mass-storage memory and faster processing. Another important point is that the number of degrees of freedom for RCS optimization algorithms is also smaller with parametric surfaces. 
- The faceting approach introduces artificial edges and vertices between facets, so that the surface presents a "faceted" appearance, while the parametric surface is smooth and conforms precisely to the real one. For that reason, the RCS computed from the faceted model contains the so-called "facet noise," with is not present when the parametric surface model is used instead.

For these reasons, parametric surfaces have been used in the GRECO code, and are also being implemented in the TOTAL code $[15,16]$, developed at the University of Cantabria, Spain.

It must be noted that the parametric-surface database is compatible with an electromagnetic analysis code for predicting the RCS of arbitrary edges. This code, which has been recently developed by our group, is based on a hybrid-mode boundary-element approach [17]

\subsection{Real-time image of the target}

Hardware graphics accelerators of high-performance workstations are able to render a $3 \mathrm{D}$ visualization of a parametric surface model in real time. Shadowed parts of the scene are removed from the image by the graphics hardware, so that the picture on the workstation screen contains only the surfaces visible from the observer's viewpoint.

As shown in Figure 2, the input information for the graphics accelerator must be, at a minimum [18], the following:

\section{- Geometric model: NURBS surface parameters}

- Rendering: Surface-reflection coefficients for specular and diffuse reflection, pattern of the reflected beam, etc.

- Illumination: Position, orientation, radiation pattern and color of the light sources

- Observer: 3D viewpoint and direction of observation

With these basic input parameters and further advanced rendering information, the result may be an extremely realistic realtime image of the aircraft, if the proper parameters are used.

It must be noted that all the rendering computations are performed separately for each one of the three $R, G, B$ color components. As shown in Figure 2, the six-dimensional output of the graphics accelerator for each pixel includes the three $R, G, B$ color

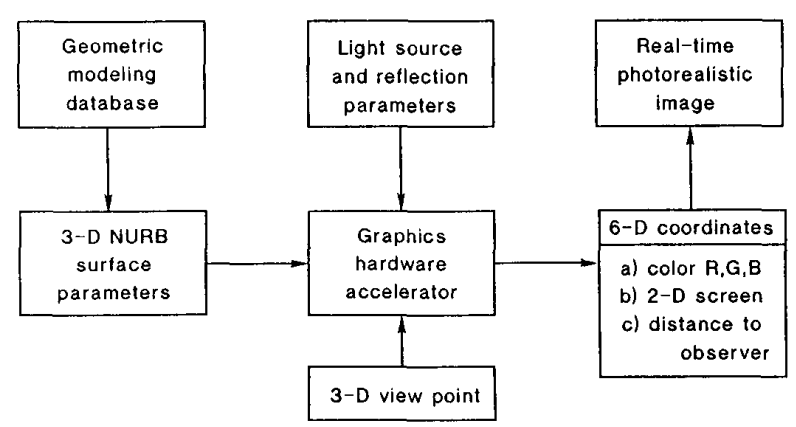

Figure 2. The inputs and outputs of the hardware graphics accelerator.

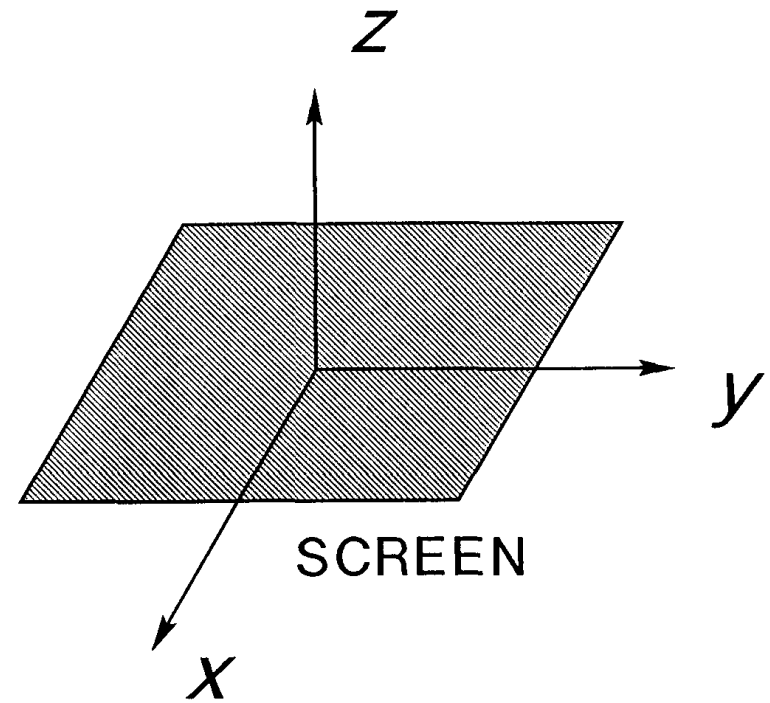

Figure 3. The three-dimensional coordinate-axis convention for graphical processing.

components; the 2D location on the screen; and the distance to the observer. For example, in the old Hewlett-Packard Turbo SRX graphics accelerator used by GRECO, the coding of this information is as follows: $2 \mathrm{D}$ screen $1280 \times 1024$ pixels; distance to the observer in 16 bits; and R,G,B color components in 8 bits each $\times 3$ $=24$ bits. However, much better resolution can be provided by the latest, more-powerful graphics accelerators.

\subsection{Graphical processing}

In this section we will use the following convention for the 3D coordinate axis (see Figure 3): $x y$ are the 2D coordinates of the workstation screen, while $z$ is along the normal direction to the screen. If the observer's viewpoint is located at the monostatic radar position, the result is that the $z$ coordinate of each pixel is equal to the distance between the observer and each surface element.

From the electromagnetic point of view, this $z$ information is of substantial importance for coherently adding the local highfrequency contribution of each surface element. Using this convention, the six-dimensional outputs of the hardware graphics accelerator are the $x, y, z, R, G, B$ coordinates and color components for each pixel.

\subsubsection{Shadowed and eclipsed surfaces identification}

As stated before, one of the main difficulties in computing the physical-optics surface integral by classical techniques (faceting approach) [2-4] is the detection of shadowed regions. However, $G R E C O$ has no need to face this problem, because hidden surfaces of the image have been previously removed by the hardware graphics accelerator.

A very simple validation of the hidden-surface elimination and of the correctness of the $z$ coordinate information, supplied by the graphics accelerator, is the two-sphere system, shown in Figure 4. The RCS computed by GRECO has been normalized with respect to the RCS of only one sphere. It can be noticed that when one of 

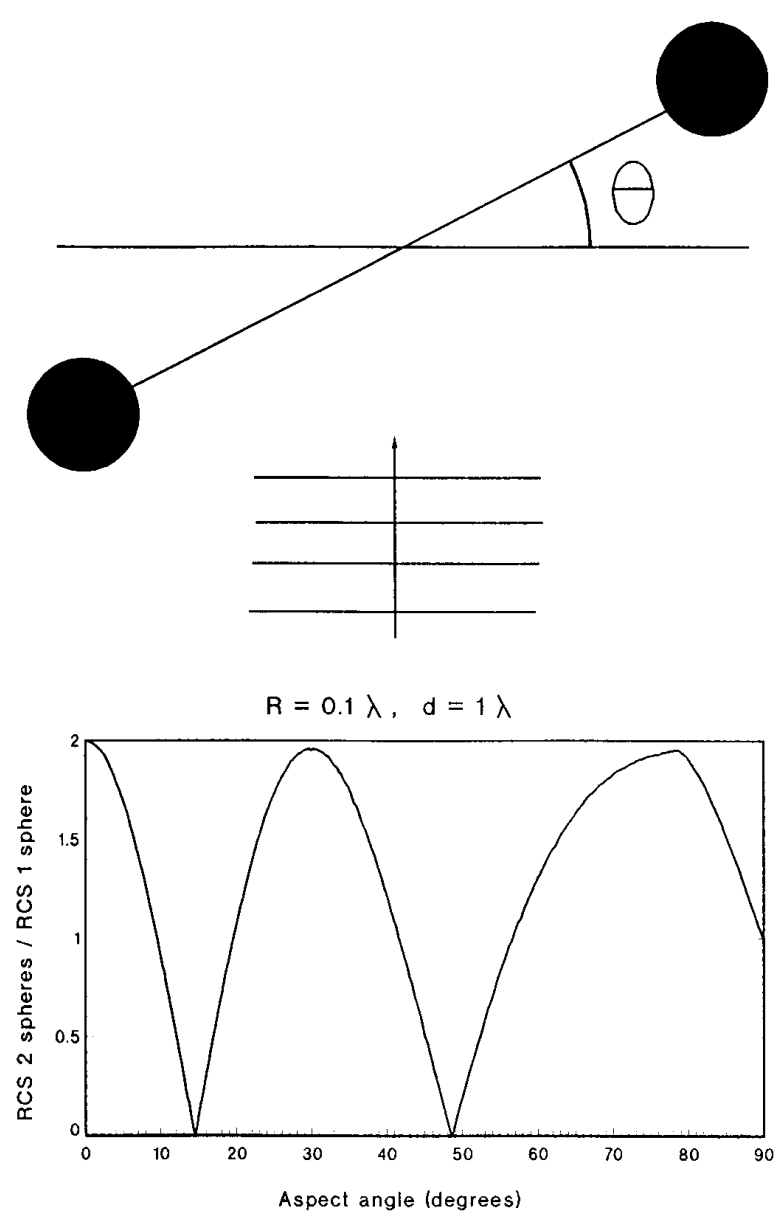

Figure 4. (a) A two-sphere system for visualizing the shadowing effects in $G R E C O$, using the hidden-surface removal capabilities of the graphics accelerator. The distance between the spheres is $\lambda$ and the radius is $0.1 \lambda$. (b) The RCS of the twosphere system is normalized with respect to the RCS of only one sphere. The shadowing effect is important when the aspect angle is close to $90^{\circ}$ : the normalized RCS of the system approaches one as the first sphere eclipses the second one.

the two spheres is shadowing the other one, with aspect angle close to $90^{\circ}$, the RCS of the two-sphere system is equal to the RCS of only the visible one.

\subsubsection{Computation of unit normal to surface}

If the scene is rendered using the Phong local-illumination model [19], the color of each pixel depends only on the normal to the surface element associated with this pixel, and on the locations of the observer and of the light sources. As the positions of both the observer and of the light sources are known, it is possible to obtain the normal to the surface for each pixel of the image from the color information.

According to the Phong illumination model, when the surface reflection is diffuse, not specular, the brightness of a pixel is computed separately for each R,G,B color as the projection, $\bar{n} \bullet r_{i}$, of the unit normal to the surface, $\bar{n}$, on the direction of illumination, $r_{i}$ (see Figure 5). For three light sources of purely green, red, and blue colors, respectively, located over each one of the three coordinate axis, the three color components for this pixel are equal to the $\left(n_{x}, n_{y}, n_{z}\right)$ components of the unit normal to surface:

$$
\begin{array}{ll}
\text { RED: } & \hat{r}_{i}=\hat{x} \Rightarrow \mathrm{R}=\hat{n} \bullet \hat{x}=n_{x} \\
\text { BLUE: } & \hat{r}_{i}=\hat{y} \Rightarrow \mathrm{B}=\hat{n} \bullet \hat{y}=n_{y} \\
\text { GREEN: } & \hat{r}_{i}=\hat{z} \Rightarrow \mathrm{G}=\hat{n} \bullet \hat{z}=n_{z} \\
& \hat{n}=\left(n_{x}, n_{y}, n_{z}\right)
\end{array}
$$

Figure 6 shows an image of F-117 stealth aircraft, illuminated according to Equation (1). Thus, a purely red color means that the unit normal to the surface is horizontal $(x)$, a purely blue color is vertical $(y)$, and a purely green color is perpendicular $(z)$, to the screen. When the unit normal is not parallel to any of the three coordinate axis, the blending of the three color components (red, blue, green) is equal to the $\left(n_{x}, n_{y}, n_{z}\right)$ components of the unit normal.

As the color components are always positive quantities, there is an ambiguity in the sign of the $\left(n_{x}, n_{y}, n_{z}\right)$ components of the unit normal. To obtain only the positive values, the graphics accelerator must display only the illuminated surfaces, with $\cos \theta_{i}>0$, and must remove the rear-facing ones, with $\cos \theta_{i}<0$. In order to obtain positive and negative values for $\left(n_{x}, n_{y}, n_{z}\right)$, it is necessary to illuminate the target from both the positive- and negative-axis directions, using different light sources. As we need a total of six light sources in order to obtain the six positive and negative values of $\left(n_{x}, n_{y}, n_{z}\right)$, and as there are only three independent color components (R,G,B), two different three-color images must be displayed separately. Figure 7 shows the two images of the generic missile, defined by $\mathrm{N}$. Youssef in [2].

\section{Electromagnetic computing}

Electromagnetic computing, the second main step in GRECO, uses as input information the $x, y, z$ coordinates and the $n_{x}, n_{y}, n_{z}$ unit normal of each illuminated pixel of the target. From the knowl-

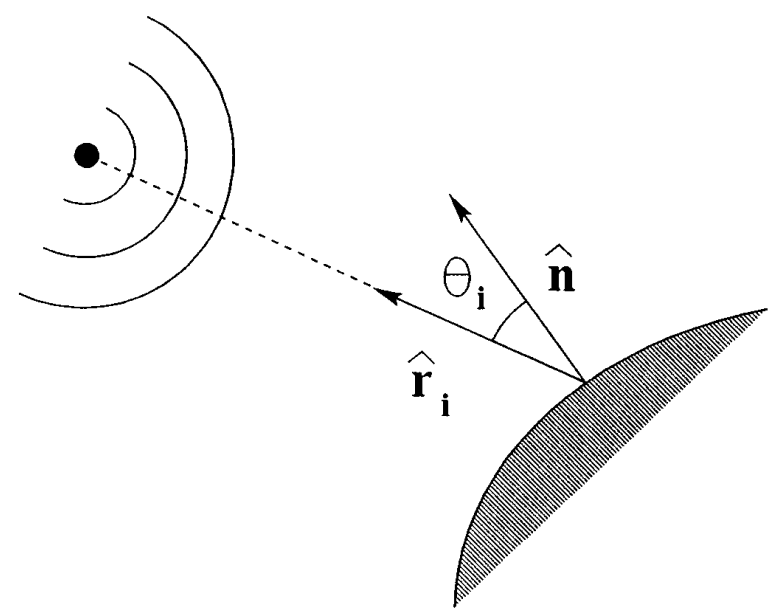

Figure 5. The geometry for diffuse reflection according to the Phong illumination model. 


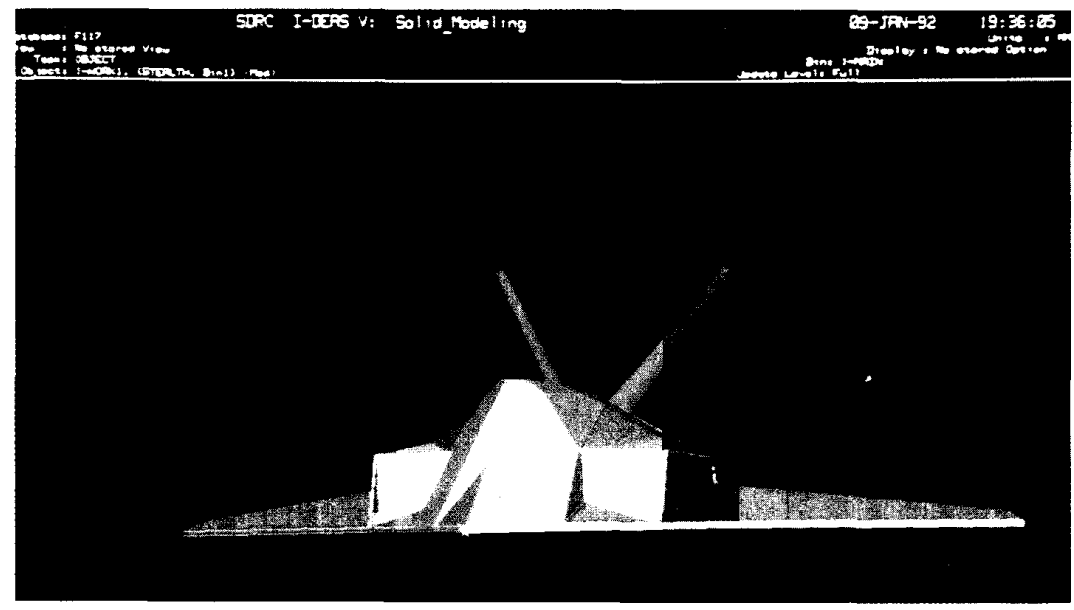

Figure 6. A rendered image of an F-117 aircraft on the workstation screen. Three red, blue, and green light sources are located on the $x, y, z$ axes, respectively. The blending of the three colors at each pixel is equal to the $\left(n_{x}, n_{y}, n_{z}\right)$ components of the unit normal.

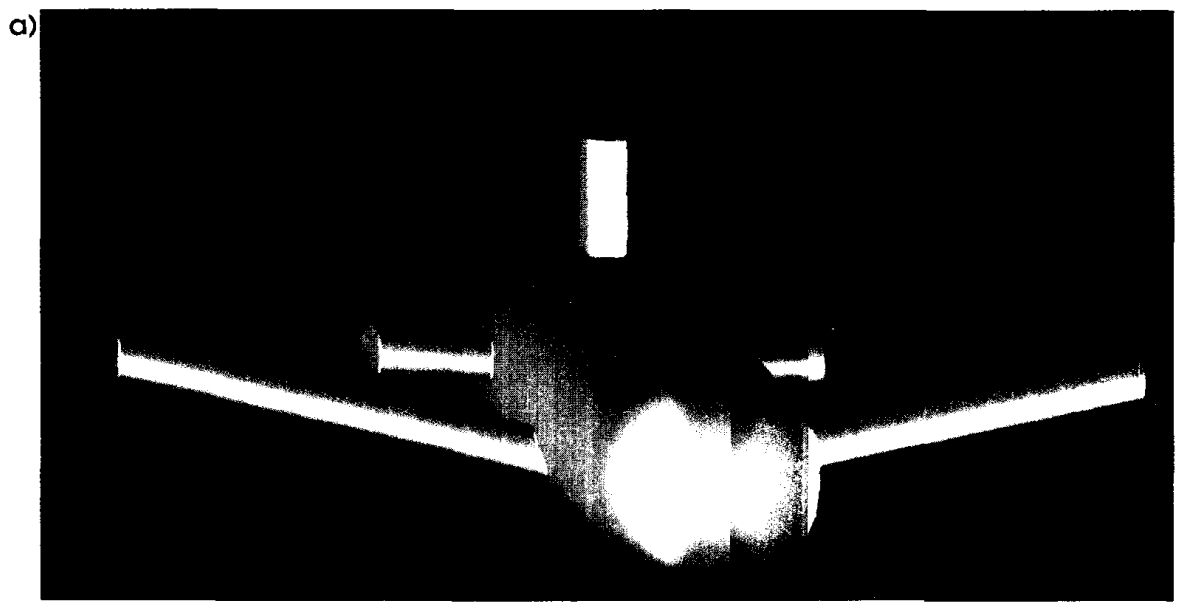

b)

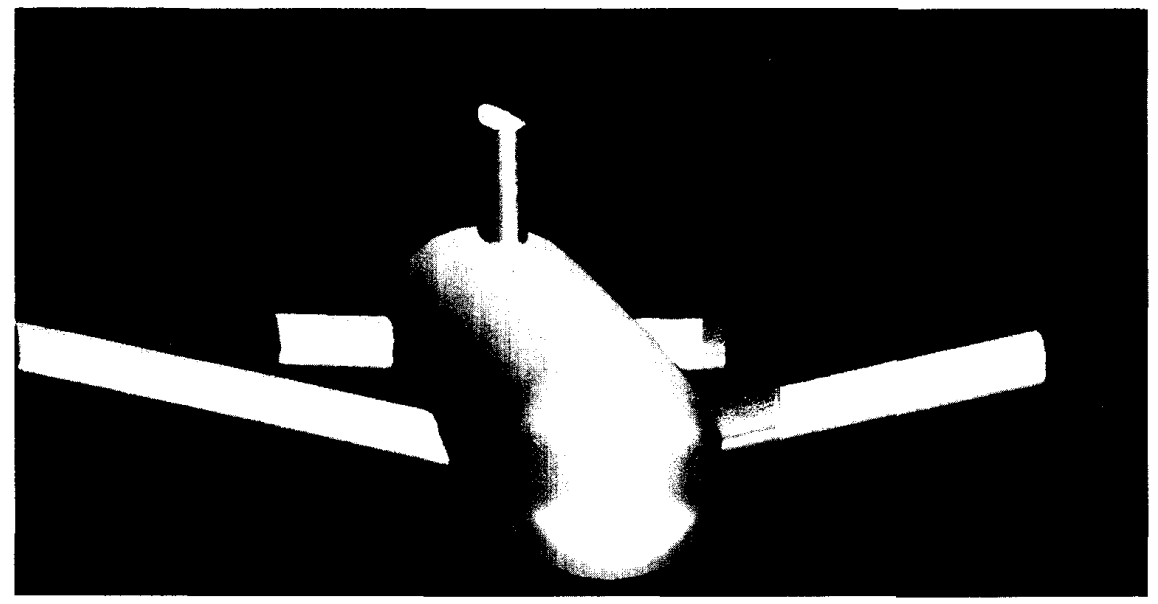

Figure 7. Rendered images of the generic missile model defined in [2]. Six red, blue, and green light sources are located on the positive and negative $x, y$ axis (a) and $y, z$ axis (b). The blending of the three colors at each pixel of the two images is equal to the positive and negative $\left(n_{x}, n_{y}, n_{z}\right)$ components of the unit normal. 
edge of this input information, a number of different high-frequency approximations can be implemented, in a way completely independent of the target geometry. As high-frequency theory for RCS prediction is well-known $[1,20]$, we will concentrate on the efficient implementation of high-frequency techniques in the GRECO code.

\subsection{Physical Optics}

According to the Physical Optics (PO) technique, the monostatic RCS of a perfectly-conducting surface can be approximated, at high frequencies, by the expression [20]

$$
\sigma=\frac{4 \pi}{\lambda^{2}}\left|\int_{s} \cos \theta \mathrm{e}^{2 j K z} d s\right|^{2}
$$

where $\theta$ is the angle between the normal to the surface and the direction of incidence, and $z$ is the distance from the differential of surface, $d s$, to the observer, projected on the direction of incidence. The surface integral extends only over the region illuminated by the incident wave.

However, the image of the target processed by GRECO is actually a projection, on the workstation screen, of the real, 3D surface (see Figure 8), so that the differential of the surface on the screen, $d s^{\prime}$, equivalent to one pixel, is equal to $d s^{\prime}=\cos \theta d s$, and the PO surface integral (2) can be written as

$$
\begin{aligned}
\sigma & =\frac{4 \pi}{\lambda^{2}} \mid \int_{s} \cos \theta \mathrm{e}^{2 j K z} d s^{2} \\
& =\frac{4 \pi}{\lambda^{2}}\left|\int_{\text {screen }} \mathrm{e}^{2 j K z} d s^{\prime}\right|^{2}
\end{aligned}
$$

Discrete computation of surface integral (3) leads to

$$
\sigma=\frac{4 \pi}{\lambda^{2}}\left|\sum_{\text {pixels }} \mathrm{e}^{2 j K z}\right|^{2}
$$

which is equal to the coherent addition of the phase contribution from all the pixels in the target image. This phase contribution is due to the distance, $z$, from each pixel to the observer.

It must be noted that Equation (4) is correct only if a pixel radiates as an infinitesimal aperture, i.e., it is equivalent to the projection on the screen of a differential of surface, $d s$, much smaller than a wavelength. If the number of pixels on the screen is large enough, this condition is usually accomplished. However, when the incidence is grazing over the surface, $\theta \rightarrow 90^{\circ}$, the projection, $d s^{\prime}$, on the screen (one pixel) is very small, but the surface, $d s$, may be very large.

Accordingly, in general we cannot assume that each pixel radiates as an infinitesimal aperture, but as one which is electrically large. Assuming that one pixel is equivalent to a rectangular aperture with uniform illumination, its contribution to the far field can be approximated by a sinc function of the angle, $\theta$, and the PO surface integral becomes, in the discrete domain,

$$
\sigma=\frac{4 \pi}{\lambda^{2}}\left|\sum_{\text {pixels }} \operatorname{sinc}\left(K \frac{\ell}{\cos \theta} \sin \theta\right) \mathrm{e}^{2 j K z}\right|^{2}
$$

where $\ell$ is the size of a square pixel on the screen, and $\ell / \cos \theta$ is the size of the $d s$ projected on this pixel.

\section{SCREEN}

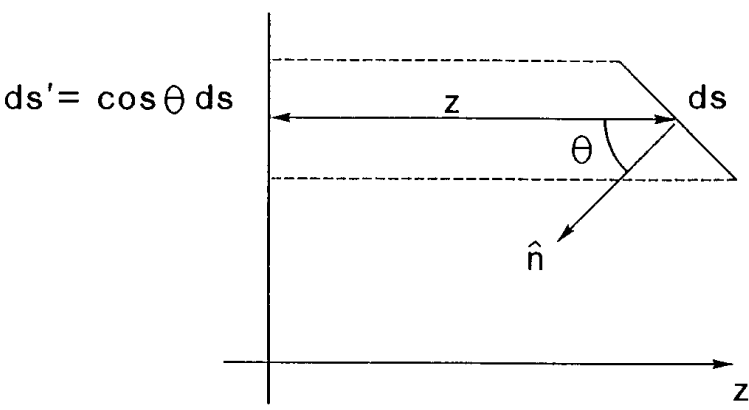

Figure 8. The surface element and its projection on the workstation screen.

Equation (5) can be implemented very efficiently in GRECO, because the sinc function depends only on the angle, $\theta$. Using the illumination sources described in Section 3.3.2 (see the F-117 in Figure 6), the green color component of each pixel is equal to

Green $=n_{z}=\cos \theta$

As the green color is codified into 8 bits, the sinc function can be tabulated in a 256-entry table, indexed by the green-color component of each pixel. If the $\mathrm{e}^{-2 j K z}$ phase exponential is also tabulated in a $2^{16}$-entry table, indexed by coordinate $z$, we can add the contribution from each pixel to the PO integral with only two floating-point real-number multiplications and additions.

\subsection{Impedance Boundary Condition}

Radar-absorbent coatings are considered through impedanceboundary-condition and physical-optics approximations, which lead to a very simple formulation of the PO surface integral. It must be noticed that the PO + IBC approach is valid only for surface reflection, when a specular reflection point exists. Impedance wedges should be treated by a higher-order approximate boundary condition.

According to the IBC, the contribution from each pixel in Equation (5) must be multiplied by the Fresnel reflection coefficients for polarizations parallel $\left(\Gamma_{\|}\right)$and perpendicular $\left(\Gamma_{\perp}\right)$ to the plane of incidence:

$$
\sigma=\frac{4 \pi}{\lambda^{2}}\left|\sum_{\text {pixels }}\left[\Gamma_{\|} E_{\|}^{i} \hat{e}_{\|}^{i}+\Gamma_{\perp} E_{\perp}^{i} \hat{e}_{\perp}^{i}\right] \operatorname{sinc}(K \ell \tan \theta) \mathrm{e}^{2 j K z}\right|^{2}
$$

where $E_{\| l}^{i}$ and $E_{\perp}^{i}$ are, respectively, the components of the incident field in the directions parallel and perpendicular to the plane of incidence, $\hat{e}_{\|}^{i}$ and $\hat{e}_{\perp}^{i}$.

Formulation of the Fresnel reflection coefficients as functions of equivalent surface impedance is well known, and can be found in $[1,20,21$, etc. $]$. The surface impedance of a dielectric coating over a perfect-conducting surface is obtained in the usual way, through a transmission-line equivalent circuit.

In order to implement Equation (7), we must first separate the incident field into its $E_{\|}^{i}$ and $E_{\perp}^{i}$ components, which is done from the knowledge of the unit normal to the surface at each pixel. As 
reflection coefficients are dependent only on the angle, $\theta$, which, according to Equation (6), is related to the green color component of each pixel in the image (see Figure 6), the parallel and perpendicular reflection coefficients can be tabulated in a 256-entry table, indexed by the green color component, thus avoiding the computation of the reflection coefficients for each pixel.

\subsection{Method of Equivalent Currents (MEC)}

According to high-frequency theory, the far field scattered from a wedge can be assumed to be that radiated by an equivalent line current, located on the edge $[1,20]$. This equivalent current depends on both the directions of incidence and of observation, relative to the orientation of the edge, so that its value is not constant along the edge. Equivalent currents are usually expressed as a function of some incremental-length diffraction coefficients [22]. The monostatic far-field scattering from the wedge, resulting from the radiation of equivalent currents, is [1]

$$
\bar{E}^{r}=E_{0} \frac{\mathrm{e}^{-j K r}}{2 \pi r} \int_{\text {edge }}\left[-D_{1} \sin \gamma \hat{e}_{\|}^{i}-D_{x} \cos \gamma \hat{e}_{\| 1}^{i}-D_{\perp} \cos \gamma \hat{e}_{\perp}^{i}\right] e^{2 j k \hat{r}-\vec{r}^{\prime}} d \ell^{\prime}
$$

where the line integral extends along the edges illuminated by the incident wave, $\hat{e}_{\|}^{i}$ and $\hat{e}_{\perp}^{i}$ are, respectively, the unit vectors parallel and perpendicular to the plane of incidence (which is defined by the incident and edge directions) and $\gamma$ is the angle between the incident electric field and $\hat{e}_{\perp}^{i}$

In Equation (8), $D_{\mid}, D_{\perp}$, and $D_{x}$ stand for the monostatic ILDCs, which depend on the angles $\alpha, \phi$, and $\beta_{r}$, defined in Figure 9. The formulation and references to the original papers for the GTD, the PTD, and Michaeli's or Mitzner's ILDC can be found in $[1,20]$. In order to compute the MEC line integral (8), the GRECO code must obtain the ILDCs for each pixel of the image laying along an edge, and must coherently add the contributions from all the pixels.

As the unit normal to both faces of the edge is known, the angles $\alpha, \phi$, and $\beta_{r}$ can be obtained using the equations [11]

$$
\begin{aligned}
& \alpha=\cos ^{-1}\left(-\hat{n}_{1} \bullet \hat{n}_{2}\right) \\
& \sin \beta_{r}=|\hat{r} \times \hat{t}|=\sqrt{t_{x}^{2}+t_{y}^{2}} \\
& \cos \phi=\frac{n_{1 x} t_{y}-n_{1 y} t_{x}}{\sqrt{t_{x}^{2}+t_{y}^{2}}}
\end{aligned}
$$

where $t$ is the unit vector along the edge direction

$$
t=\frac{\hat{n}_{1} \times \hat{n}_{2}}{\left|\hat{n}_{1} \times \hat{n}_{2}\right|}
$$

As the reflection at the (curved or flat) faces of the wedge has been already obtained in Section 4.1 using Physical Optics, we now must compute only the contribution to far-field scattering from the edge alone. Although the exact ILDCs for scattering from edges without surface reflection at faces are Mitzner ILDCs, in the GRECO code we have implemented Physical Theory of Diffraction coefficients. The reason for this is that in monostatic scattering, PTD coefficients are equal to Mitzner ILDCs, except for the assumption that there are no cross-polarization effects when the

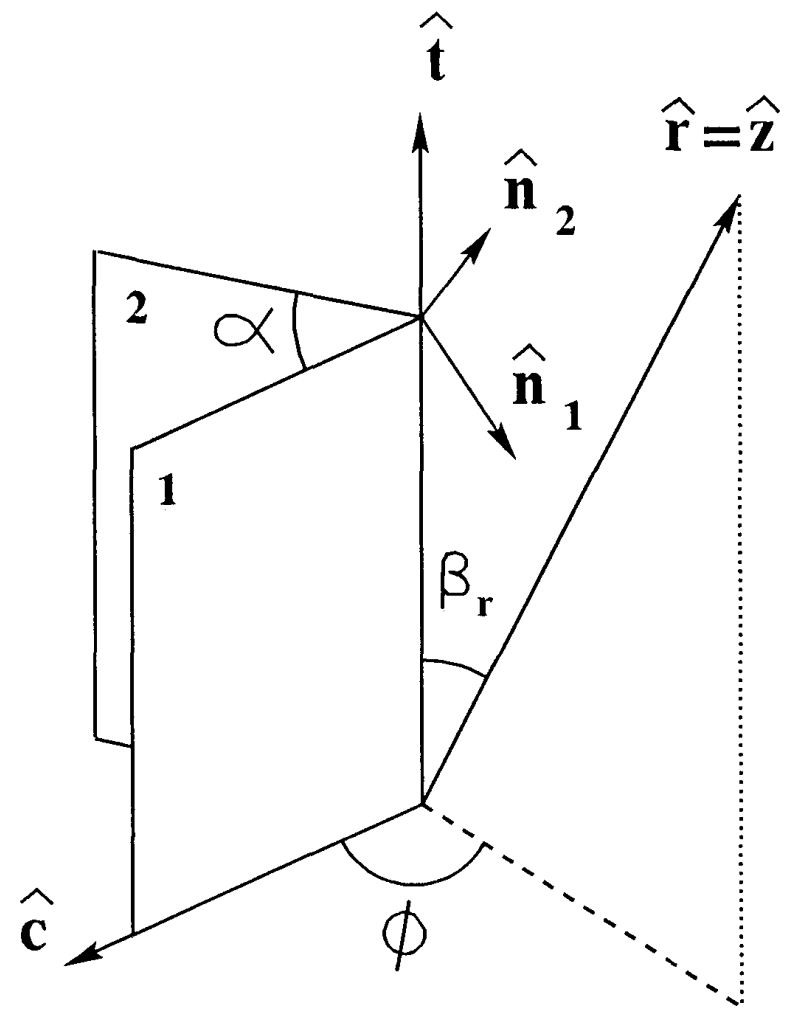

Figure 9. The wedge geometry. The directions of incidence and observation are along the $z$-axis, as defined in Figure 3.

incident field is perpendicular to the plane of incidence, i.e. $D_{x}=0$. This is actually a very good approximation when the incidence direction is near the plane perpendicular to the edge, and it is wellknown that normal incidence produces the RCS flashes from the edge scattering.

In summary, the GRECO code computes high-frequency scattering from edges by the Method of Equivalent Currents in the following steps:

1. An image of the target is made on the workstation screen. Hidden edges are removed by the graphics accelerator hardware, so that only visible ones are displayed.

2. The surface unit normal at each pixel of the image is computed by graphical processing of the image (see 3.3.2)

3. Edges are detected on the target image as discontinuities of the unit normal to the surface.

4. For each pixel along the detected edges, $\alpha, \phi$, and $\beta_{r}$ are computed from the unit normal to each face of the wedge, using Equations (9)-(12). Monostatic PTD diffraction coefficients are then obtained, using a very simple linear approximation, which saves running time by avoiding the computation of trigonometric functions.

5. The line integral (Equation 8) of the MEC is evaluated, coherently adding the PTD coefficients for each pixel. 


\section{Results for complex objects}

The graphical electromagnetic computing technique presented in this paper has been validated by comparing the results for simple and canonical objects with analytical solutions [11]. Results for complex radar targets can be found in [6-11], and show good agreement with both measurements and faceting-approach codes.

In this section, we will only present the results obtained by GRECO for the generic missile, defined by N. Youssef in his classic paper [2], together with the result for the airfoil section defined at the JINA' 90 workshop [23].

Using an old Hewlett-Packard 380 workstation (a Motorola 68040 CPU with a speed of only 2.5 MFlops) and a Turbo SRX graphics accelerator, we can compute the RCS of a complex aircraft in 0.2 seconds/angle (PO), or about 5 to 10 seconds/angle (MEC). This speed should be improved by a factor of 5 or 10 using the new and faster HP-700 RISC workstations with the Turbo VGX graphics accelerator.

\subsection{Generic Missile}

Figure 10 shows the geometry of the generic missile defined in [2]. The results of the faceting-approach code RECOT'A, developed by Boeing Aerospace, can be found in [2]. Figure 11 presents the results of GRECO (only Physical Optics), compared with the prediction of the TOTAL code $[3,4]$, developed at the University of Cantabria, Spain. The TOTAL code is based on a facets-andNURBS model, and takes into account surface reflection, edge diffraction, and surface-surface and surface-edge interactions

The following table compares the monostatic RCS results of GRECO, TOTAL, and RECOTA, with measurements performed by Boeing Aerospace [2] at a frequency of $12 \mathrm{GHz}$, and with vertical polarization. The agreement between the three predictions and the measurements is good, except for the flash produced by the leading

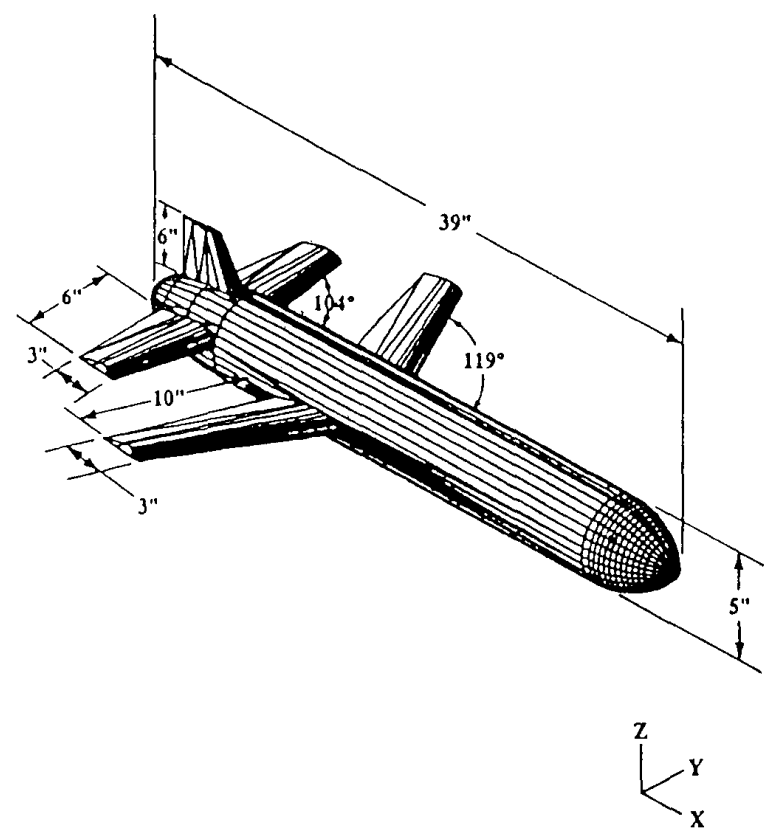

Figure 10. The generic missile model defined in [2]. The fuselage length is about $40 \lambda$ at $12 \mathrm{GHz}$.

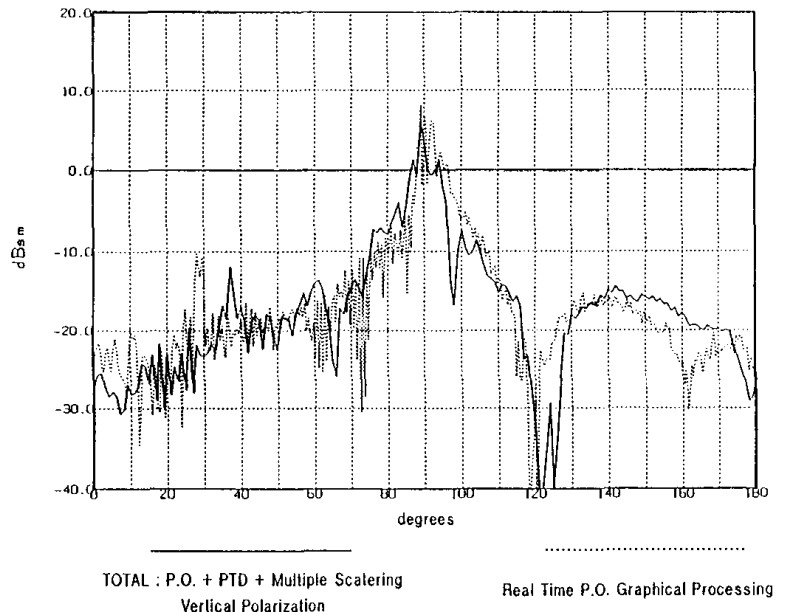

Figure 11. The results of GRECO (only Physical Optics) compared with the predictions of TOTAL $[3,4]$ at $12 \mathrm{GHz}$.

\begin{tabular}{|l|c|c|c|c|}
\hline & Measured & RECOTA & TOTAL & GRECO \\
\hline \hline Leading edge & $16^{\circ}$ & $13^{\circ}$ & & $11^{\circ}$ \\
horiz. stabil. & $-19 \mathrm{dBsm}$ & $-17 \mathrm{dBsm}$ & & $-21 \mathrm{dBsm}$ \\
\hline $\begin{array}{l}\text { Leading edge } \\
\text { of wing }\end{array}$ & $29^{\circ}$ & $29^{\circ}$ & $37^{\circ}$ & $29^{\circ}$ \\
\hline $\begin{array}{l}\text { Fuselage and } \\
\text { vert. stabil. }\end{array}$ & $8 \mathrm{dBs}$ & $-12 \mathrm{dBsm}$ & $-12 \mathrm{dBsm}$ & $-11 \mathrm{dBsm}$ \\
\hline RCS null & $124^{\circ}$ & $90^{\circ}$ & $89^{\circ}$ & $91^{\circ}$ \\
\hline RCS angles & $-18 \mathrm{dBsm}$ & $-20 \mathrm{dBsm}$ & $-15 \mathrm{dBsm}$ & $8 \mathrm{dBsm}$ \\
\hline $140^{\circ}-150^{\circ}$ & & & $122^{\circ}-125^{\circ}$ & $119^{\circ}$ \\
\hline
\end{tabular}

edge of the wing in the TOTAL prediction. The reason for this discrepancy is an error in the missile geometric model used by the TOTAL code.

Physical Optics results of GRECO agree well with measurements because the vertical-polarization diffraction at the trailing edges is negligible. In general, we have noticed that the first-order PO approximation usually predicts the RCS of non-stealth radar targets (for example, the Boeing 727 [6-11]) with reasonable accuracy, so that the computation of edge diffraction and multiple interactions is not always necessary.

\subsection{Airfoil section}

Figure 12 shows the two-dimensional airfoil-section geometry, as defined in the workshop, "RCS of Perfectly- Conducting or Coated Bodies," [23] held at Nice in November, 1990. In Figures 13 and 14, the GRECO high-frequency prediction is compared with a numerical-method solution, presented at the workshop by the Centre Commun de Rech. Louis-Bleriot of AEROSPATIALE.

It can be noticed, in Figures 13 and 14, that PO results correctly predict surface reflection, but not edge diffraction, when the incident polarization is parallel to the edge (TM). However, if the Method of Equivalent Currents with PTD coefficients is added to PO, the result agrees very well with the numerical solution. 


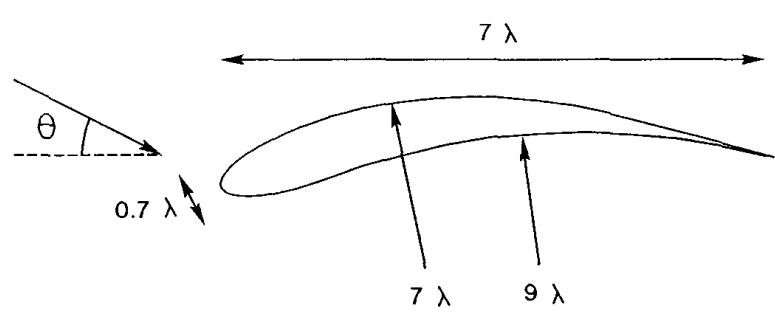

Figure 12. The airfoil section defined in the JINA'90 workshop [23].

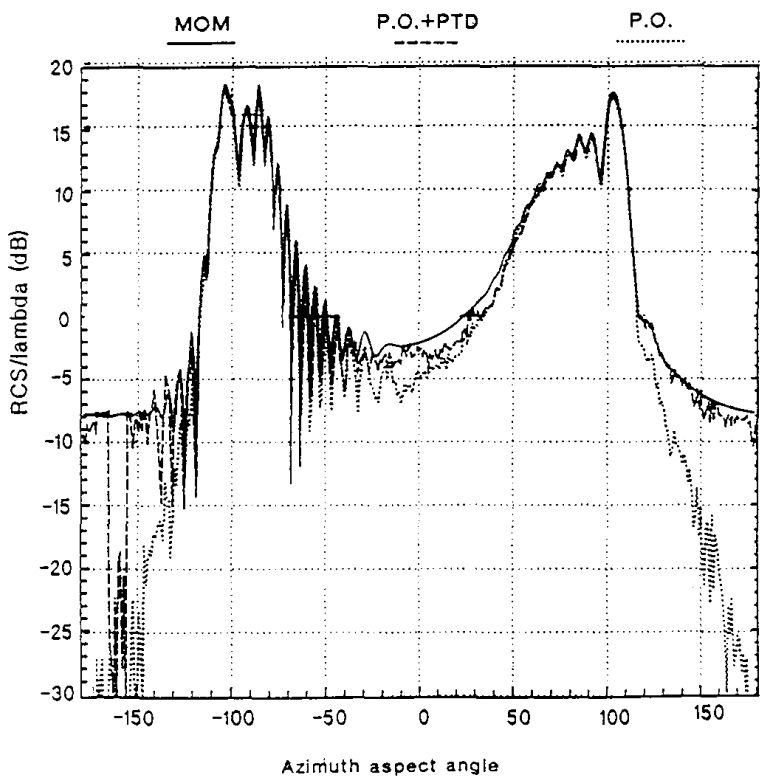

Figure 13. The GRECO results for the Airfoil in Figure 12, for TM polarization (Physical Optics and Physical Optics + Physical Theory of Diffraction), compared with a numerical solution [23].

\section{Conclusions}

In this paper we have presented a new implementation of well-known high-frequency techniques. Using a 3D workstation with a graphics hardware accelerator, monostatic RCS prediction is obtained in real time for large and complex radar targets

Graphical electromagnetic computing (GRECO) has the following advantages over classical techniques [2-4]

- The target can be modeled by parametric NURB surfaces, requiring less mass-storage memory that the faceting approach, and enabling more accurate adjustment to the real target surface, thus avoiding the "facet noise" usually present in classical facetmodeling codes.

- The hardware graphics accelerator removes hidden surfaces and edges, so that they do not contribute to surface or line integrals. The difficult and timeconsuming software identification of shadowed

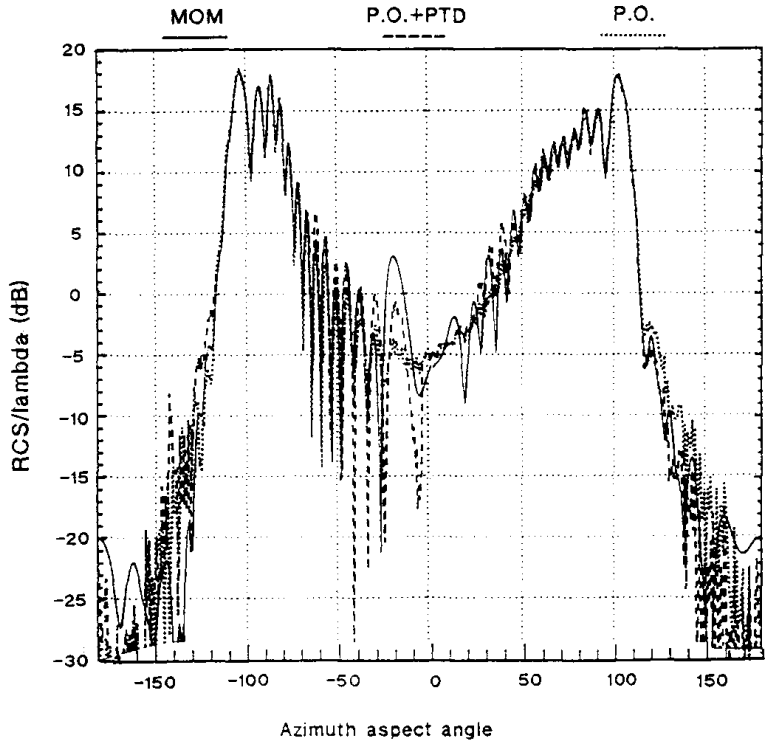

Figure 14. The GRECO results for the Airfoil in Figure 12, for TE polarization (Physical Optics and Physical Optics + Physical Theory of Diffraction), compared with a numerical solution [23].

regions is avoided, which leads to a great advantage over the classical codes for RCS prediction.

- The surface and line integrals (PO and MEC) are evaluated by graphical processing of an image of the target on the workstation screen. As this is independent of target complexity and electrical size, the CPU time and RAM requirements do not increase with target size or complexity.

- The graphical processing approach obtains a $2 \mathrm{D}$ matrix, containing the unit normal to the surface over the illuminated regions of the target. As this is independent to the electromagnetic part of the computation, it is easy to develop and integrate code for computing the different high-frequency approximations This electromagnetic code is relatively small in size, and absolutely independent of the target geometry.

- The approach permits real-time computation with a high-performance workstation and hardware graphics accelerator, while the classical techniques require powerful super computers in order to obtain real-time results

- The GRECO code can be integrated with a CAD geometric modeling package [12], thus providing an efficient tool for interactive modeling, design and analysis of aircraft with RCS specifications (see Section 7)

It must be noted that GRECO is able to analyze targets of electrical size as large as $2^{n} / 16 \lambda$, with a maximum phase error of $\lambda / 8$, where $n$ is the number of bits in which the distance, $z$, to the observer is discretized. This means that we can analyze $4000 \lambda$ with the usual 16-bit discretization. The resolution in the discretization of $x y$ 2D screen coordinates, usually $1024 \times 2048$ pixels, only limits 
the maximum complexity of the target (details must be larger than a pixel), but does not limit the target electrical size, because $x, y$ lay on a plane perpendicular to the directions of incidence and observation.

In conclusion, graphical processing is probably the optimum approach for analyzing very large and complex aircraft, using highfrequency approximations. In Section 7, the application to interactive design of aircraft with RCS specifications will be discussed.

However, there are some scattering sources that cannot be analyzed by GRECO: cavities at engine inlets, creeping waves, discontinuities and slots over the aircraft surface, etc. These effects should be analyzed by different codes, and the results added to GRECO.

\section{Potential application to RCS optimization}

As the GRECO code is based on graphical processing of an image of the target, it can be easily integrated with the CAD software package [12] used for modeling the aircraft. This makes possible the interactive modeling, design, and analysis of aircraft with RCS specifications.

Moreover, the GRECO code has some other important advantages for automatic RCS minimization, using non-linear constrained optimization methods:

- Parametric surface modeling with NURBS allows a very large reduction in the number of parameters necessary to define the surface, which may accelerate the optimization run-time by several orders of magnitude.

- The sensitivity analysis of the RCS optimization algorithm is simplified, because specular-reflection points on the target surface are visible on the workstation screen. This means that the parameters to which the RCS is more sensitive are the local control points of the specular surface, so that the number of degrees of freedom to optimize is reduced from of the order of thousands to of the order of tens.

- 3D visualization of the target allows the monitoring of shape evolution in real time.

\section{Acknowledgments}

This work has been supported by the Spanish "Comision Interministerial de Ciencia y Tecnologia" (CICYT) under the project ACCION ESPECIAL PRONTIC "Programa de investigación aplicada para el desarrollo y validación de métodos de cálculo numérico para la predicción y análisis de las características de los ecos radar (RCS) y su reducción," TIC 88-288E. Management: "Dirección General de Telecomunicaciones" (D.G.Tel). Coordination: C.A.S.A.

\section{References}

1. E. F. Knott, J. F. Shaeffer, M. T. Tuley, Radar Cross Section, Artech House, 1985.

2. N. N. Youssef, "Radar Cross Section of Complex Targets," Proc. IEEE, Vol. 77, No. 5, May 1989.

16
3. R. Abad, J. I. Casado, P. L. Primo del Val, R. Torres, M. Domingo, F. Rivas, M. F. Cátedra, "Modelos matem ticos para el estudio de la RCS debida a doble reflexión, difracción en aristas, y sombras por eclipse en parches poligonales planos," in $V$ Symposium Nacional del Comité Español de la URSI, pp. 190-194, Vigo, 26-28th Septiember de 1990.

4. M. Domingo, F. Rivas, M. F. Cátedra, R. Abad, J. I. Casado, P. L. Primo del Val, R. Torres, "Programa base de GTD-PTD para el cálculo de la RCS de cuerpos conductores modelados por parches planos y teniendo en cuenta simple y doble reflexión, difracción en aristas y eliminación de zonas oclutas," in $V$ Symposium Nacional del Comité Español de la URSI, pp. 185-189, Vigo, 26-28th Septiember 1990.

5. W. B. Gordon, "Far Field Approximation of the KirchhoffHelmholtz Representation of Scattered Fields," IEEE Trans. Ant. Prop., AP-23, No. 5, July 1975, pp. 864-876.

6. J. M. Rius, M. Ferrando, "Real Time Radar Cross-Section of Complex Targets by Physical Optics Graphical Processing," in Digest 1990 IEEE International Symposium on Antemas and Propagation," pp. 1280-1283, Dallas, 7-11 May 1990.

7. J. M. Rius, M. Vall-llossera, M. Ferrando, "Fast Algorithms for Radar Cross Section Computation of Complex Objects," in Journées Internationales de Nice sur les Antennes JINA '90, Nice, France, 13-16th Noviember 1990.

8. J. M. Rius, M. Vall-1lossera, "High Frequency Radar Cross Section of Complex Objects in Real Time," in Digest 1991 IEEE AP-S International Symposium, London, Ontario, Canada, June 1991.

9. J. M. Rius, M. Vall-llossera, A. Cardama, "High Frequency RCS of Perfectly Conducting or Coated Complex Objects in Real Time," in 21th European Microwave Conference, 9-12th September, 1991, Stuttgart, Germany.

10. J. M. Rius, M. Vall-llossera, A. Cardama, "Real Time RCS of Perfectly Conducting or Coated Radar Targets," in 2nd International Conference on Electromagnetics in Aerospace Applications (ICEAA 91), 17-20th September 1991, Torino, Italy.

11. J. M. Rius, "Sección Recta de Blancos Radar Complejos en Tiempo Real," Tesis Doctoral, Universidat Politècnica de Catalunya, July 1991.

12. "I-DEAS Geomod Users Guide," Structural Dynamics Research Corporation (SDRC), Milford, OH 45150.

13. C. de Boor, A Practical Guide to Splines," New York, Springer-Verlag, 1978.

14. G. Farin, Curves and Surfaces for Computer Aided Geometric Design: A practical Guide," New York, Academic Press, 1988.

15. M. Pizarroso, J. Pérez, M. F. Cátedra, "Software geométrico para el cálculo de RCS de sólidos modelados por NURBS," in $V$ Symposium Nacional del Comité Español de la URSI, pp. 195-199, Vigo, 26-28th September 1990

16. X. Fernández Hermida, A. García Pino, "Spline Modeling to Compute the RCS of Arbitrarily Shaped Cavities by the GO/AI Method," in Digest of IEEE/URSI Meeting, Dallas, 7-11 May 1990, p. 280. 
17. J. M. Alvarez, J. C. Cruellas, M. Ferrando, "A Hybrid ModalBoundary Element Method for Electromagnetic Scattering from Arbitrary Conducting Wedges," in Digest of 1990 IEEE International Symposium on Antennas and Propagation," pp. 1288-1291, Dallas, 7-11 May 1990

18. B. R. Dewey, "Computer Graphics for Engineers," New York: Harper \& Row, 1988.

19. B. T. Phong, "Illumination for Computer Generated Images," $\mathrm{PhD}$ Dissertation, University of Utah, 1973.

20. A. L. Maffet, Topics for a Statistical Description of Radar Cross Section," New York: John Wiley \& Sons, 1989.
21. D. Klement, J. Peissner, V. Stein, "Special Problems in Applying the Physical Optics Method for Backscatter Computations of Complicated Objects," IEEE Trans. Ant. Prop., AP-36, No. 2, February 1988 , pp. 228-237.

22. R. A. Shore, A. D. Yaghjian, "Incremental Diffraction Coefficients for Planar Surfaces," IEEE Trans. Ant. Prop., AP-36, No. l, January 1988

23. Workshop organized by Dassault Aviation, Société Mothesim, and CNET-PAB La Turbie, Nice, France, 16th Noviember 1990.

\section{Nosich Conference Grant Awardees Announced}

The first two recipients of the Nosich Conference Grant have been announced: Alexander Y. Svezhentsev, and Andrey Andrenko. The Noisch Conference Grant is awarded to young $\mathrm{PhD}$ candidates or recipients who are from the ex-USSR, for the purpose of attending international conferences held outside the ex-USSR. An announcement of the Grant, and further details on its requirements, appears in this issue of the Magazine.

A Grant was given to Svezhentsev for attending the International Conference on Lightwave Technology and Communications (BILCON'92), held at Bilkent University, Ankara, Turkey, July 26-28, 1992. He presented a paper entitled, "Microstrip/Slot Lines with Optically Switched Characteristics." He also received a partial award to attend the International Symposium on Antennas and Propagation (ISAP'92), held in Sapporo, Japan, September 22-25, where be presented a paper entitled, "Coupling Effects for Complex Waves in Multilayer Cylindrical Strip and Slot Lines."

A. Y. Svezhentsev was born in 1957 in Tambov, Russia. He obtained both the MS and $\mathrm{PhD}$ degrees in Radiophysics from the Kharkov University, Ukraine, in 1979 and 1987, respectively. He is currently a Senior Researcher with the Institute of Radiophysics \& Electronics, Ukrainian Academy of Sciences (IRE USE), Kharkov. His main research interests are microstrip and slot lines, open waveguides, and complex-mode behavior.

Andrenko also received a Grant to attend BILCON'92, where he presented a paper entitled, "Wave Transformation by Integrated Finite-Periodic Metal-Rod Coupler in a Thin-Film Guide." He also received a partial Grant to attend the URSI Symposium on EM Theory in Sydney, Australia, August 17-20, where he presented a paper entitled, "Rigorous Solution of the Problem of Dielectric Slab Mode Scattering by Finite-Periodic Inhomogeneities."

A. S. Andrenko was born in 1964 in Kharkov, Ukraine. He obtained both the MS and $\mathrm{PhD}$ degrees in Radiophysics from the Kharkov University in 1986 and 1992 , respectively. He is currently a Junior Researcher with the IRE UAS. His main research interests are scattering from inhomogeneities in dielectric-slab waveguides.

\section{THE NOSICH CONFERENCE GRANT}

\section{Electromagnetic Theory and Applications}

Ex-USSR applicants are welcome to apply for the annual Conference Grant for attending international conferences held outside the ex-USSR. The scope of the Conference must be within the topic areas of Electromagnetic Wave Theory and Applications, such as Antennas, Waveguides, Scattering, etc. Applicants should 1) be under 40,2 ) have completed or be in the process of completing a $\mathrm{PhD}$ (Candidate of Science) thesis in radiophysics, and 3 ) have good oral and written English skills. Preference is given to young researchers of the Ukraine traveling to the West for the first time. However, applicants from other countries are eligible.

The amount of Grant depends on circumstances, but does not exceed \$500 USD. The Grant is not restricted to travel expenses, but can cover registration fees and accommodations. There is no application deadline. However, application should be made well before a proposed event. It should be written in English, in free format, and should contain references. A document certifying the acceptance of the conference paper must be enclosed. Normally, the successful applicant is supposed to pass a personal interview. Correspondence should be sent to both addresses:

Prof. A. I. Nosich

Visiting Scientist

Dept. of Electrical Engineering and Computer Science

Kumamoto University

Kumamoto 860, Japan

Tel: (81) 963442111 ext. 3635

Fax: (81) 9634515533635

Fax: (81) 963451553
Prof. A. I. Nosich, c/o Dept. Sci. Techn. Information Institute of Radiophysics and Electronics

Ukrainian Academy of Sciences

12, Ul. Proskury, Kharkov,

310085, Ukraine

Tel: (0572)-448486

Fax: (0572)-441105 